JOURNAL OF THE

AMERICAN MATHEMATICAL SOCIETY

Volume 17, Number 1, Pages 181-196

S 0894-0347(03)00438-7

Article electronically published on October 3, 2003

\title{
POISSON BRACKETS AND TWO-GENERATED SUBALGEBRAS OF RINGS OF POLYNOMIALS
}

IVAN P. SHESTAKOV AND UALBAI U. UMIRBAEV

\section{INTRODUCTION}

Let $A=F\left[x_{1}, x_{2}, \ldots, x_{n}\right]$ be a ring of polynomials over a field $F$ on the variables $x_{1}, x_{2}, \ldots, x_{n}$. It is well known (see, for example, [11]) that the study of automorphisms of the algebra $A$ is closely related with the description of its subalgebras. By the theorem of P. M. Cohn [4, a subalgebra of the algebra $F[x]$ is free if and only if it is integrally closed. The theorem of A. Zaks 13 says that the Dedekind subalgebras of the algebra $A$ are rings of polynomials in a single variable. A. Nowicki and M. Nagata 8 proved that the kernel of any nontrivial derivation of the algebra $F[x, y], \operatorname{char}(F)=0$, is also a ring of polynomials in a single generator. An original solution of the occurrence problem for the algebra $A$, using the Groebner basis, was given by D. Shannon and M. Sweedler [9]. However, the method of the Groebner basis does not give any information about the structure of concrete subalgebras. Recall that the solubility of the occurrence problem for rings of polynomials over fields of characteristic 0 was proved earlier by G. Noskov [7].

The present paper is devoted to the investigation of the structure of twogenerated subalgebras of $A$. In the sequel, we always assume that $F$ is an arbitrary field of characteristic 0 . Let us denote by $\bar{f}$ the highest homogeneous part of an element $f \in A$, and by $\left\langle f_{1}, f_{2}, \ldots, f_{k}\right\rangle$ the subalgebra of $A$ generated by the elements $f_{1}, f_{2}, \ldots, f_{k} \in A$.

Definition 1. A pair of polynomials $f_{1}, f_{2} \in A$ is called $*$-reduced if they satisfy the following conditions:

1) $\bar{f}_{1}, \bar{f}_{2}$ are algebraically dependent;

2) $f_{1}, f_{2}$ are algebraically independent;

3) $\bar{f}_{1} \notin\left\langle\bar{f}_{2}\right\rangle, \bar{f}_{2} \notin\left\langle\bar{f}_{1}\right\rangle$.

Recall that a pair $f_{1}, f_{2}$ with condition 3 ) is usually called reduced. Condition 1 ) means that we exclude the trivial case when $\bar{f}_{1}, \bar{f}_{2}$ are algebraically independent. We do not consider the case when $f_{1}, f_{2}$ are algebraically dependent. Concerning this case, recall the well-known theorem of S. S. Abhyankar and T. -T. Moh [1], which says that if $f, g \in F[x]$ and $\langle f, g\rangle=F[x]$, then $\bar{f} \in\langle\bar{g}\rangle$ or $\bar{g} \in\langle\bar{f}\rangle$.

Received by the editors January 8, 2003

2000 Mathematics Subject Classification. Primary 13F20, 13P10; Secondary 14R10, 14R15, $17 \mathrm{~B} 63$.

Key words and phrases. Rings of polynomials, Poisson brackets, subalgebras.

The first author was supported by CNPq.

The second author was supported by the FAPESP Proc. 00/06832-8. 
The main result of this paper, formulated in Theorem 3, gives a lower bound for degrees of the elements of the subalgebra $\left\langle f_{1}, f_{2}\right\rangle$, where $f_{1}, f_{2}$ is a $*$-reduced pair. In particular, this estimate yields a new proof of the theorem of H. Jung [5] on automorphisms of the rings of polynomials in two variables. The estimate involves a certain invariant of the pair $f_{1}, f_{2}$ which depends on the degrees of $f_{1}, f_{2}$ and of their Poisson bracket.

The paper is structured as follows. In Section 2, we imbed the algebra $A$ into the free Poisson algebra on the same set of variables. In this way, we introduce a Poisson bracket on $A$ as a restriction of the bracket in the free Poisson algebra. We investigate elementary properties of the Poisson bracket in $A$ and prove for it an analogue of G. M. Bergman's theorem [3] on centralizers (Theorem 1). In Section 3 , the structure of subalgebras of $A$ generated by a $*$-reduced pair $f_{1}, f_{2}$ with the condition $\operatorname{deg}\left[f_{1}, f_{2}\right]>\min \left(\operatorname{deg} f_{1}, \operatorname{deg} f_{2}\right)$ is investigated in detail, and a lower bound for the degrees of the elements of such subalgebras is obtained (Theorem 2 ). The main result is deduced from Theorem 2 in Section 4, where some relevant examples of two-generated subalgebras are also given.

\section{POISSON BRACKETS}

First, we recall the definition of Poisson algebras (see [10]).

Definition 2. A vector space $B$ over a field $F$ endowed with two bilinear operations $x \cdot y$ (a multiplication) and $[x, y]$ (a Poisson bracket) is called a Poisson algebra, if $B$ is a commutative associative algebra under $x \cdot y, B$ is a Lie algebra under $[x, y]$, and $B$ satisfies the following identity (the Leibniz identity):

$$
[x \cdot y, z]=[x, z] \cdot y+x \cdot[y, z] .
$$

An important class of Poisson algebras is given by the following construction. Let $L$ be a Lie algebra with a linear basis $l_{1}, l_{2}, \ldots, l_{k}, \ldots$. Denote by $P(L)$ the ring of polynomials on the variables $l_{1}, l_{2}, \ldots, l_{k}, \ldots$ The operation $[x, y]$ of the algebra $L$ can be uniquely extended to a Poisson bracket $[x, y]$ on the algebra $P(L)$ by means of formula (11), and $P(L)$ becomes a Poisson algebra [10].

Now let $L$ be a free Lie algebra with free generators $x_{1}, x_{2}, \ldots, x_{n}$. Then $P(L)$ is a free Poisson algebra on the same set of generators [10. We will denote this algebra by $P L\left\langle x_{1}, x_{2}, \ldots, x_{n}\right\rangle$. If we choose a homogeneous basis

$$
x_{1}, x_{2}, \ldots, x_{n},\left[x_{1}, x_{2}\right], \ldots,\left[x_{1}, x_{n}\right], \ldots,\left[x_{n-1}, x_{n}\right],\left[\left[x_{1}, x_{2}\right], x_{3}\right], \ldots
$$

of the algebra $L$ with nondecreasing degrees, then $P L\left\langle x_{1}, x_{2}, \ldots, x_{n}\right\rangle$, as a vector space, coincides with the algebra of polynomials on these elements. Evidently, the vector space $P L\left\langle x_{1}, x_{2}, \ldots, x_{n}\right\rangle$ is graded by degrees on $x_{i}$, and for every element $f \in P L\left\langle x_{1}, x_{2}, \ldots, x_{n}\right\rangle$, the highest homogeneous part $\bar{f}$ and the degree function $\operatorname{deg} f$ can be defined in an ordinary way. Note that

$$
\overline{f g}=\bar{f} \bar{g}, \quad \operatorname{deg}(f g)=\operatorname{deg} f+\operatorname{deg} g, \quad \operatorname{deg}[f, g] \leq \operatorname{deg} f+\operatorname{deg} g .
$$

It is natural to identify the ring of polynomials $A=F\left[x_{1}, x_{2}, \ldots, x_{n}\right]$ with the subspace of the algebra $P L\left\langle x_{1}, x_{2}, \ldots, x_{n}\right\rangle$ generated by the elements

$$
x_{1}^{r_{1}} x_{2}^{r_{2}} \ldots x_{n}^{r_{n}}, \quad r_{i} \geq 0,1 \leq i \leq n .
$$

Put also

$$
C=\bigoplus_{1 \leq i<j \leq n}\left[x_{i}, x_{j}\right] A \subseteq P L\left\langle x_{1}, x_{2}, \ldots, x_{n}\right\rangle
$$


The next lemma follows immediately from identity (11).

Lemma 1. If $f, g \in A$, then

$$
[f, g]=\sum_{1 \leq i<j \leq n}\left[x_{i}, x_{j}\right]\left(\frac{\partial f}{\partial x_{i}} \frac{\partial g}{\partial x_{j}}-\frac{\partial g}{\partial x_{i}} \frac{\partial f}{\partial x_{j}}\right) \in C .
$$

Thus, the Poisson bracket on $P L\left\langle x_{1}, x_{2}, \ldots, x_{n}\right\rangle$ defines a mapping

$$
[\cdot, \cdot]: A \times A \longrightarrow C, \quad(x, y) \mapsto[x, y] .
$$

It follows immediately from (11) that, for any $f \in A$, the mapping

$$
\operatorname{ad}(f): A \longrightarrow C, \quad x \mapsto[x, f],
$$

is a derivation of the algebra $A$ with coefficients in the free $A$-module $C$ (see [11]).

For any $f \in A$ we put also

$$
\partial f=\left(\frac{\partial f}{\partial x_{1}}, \frac{\partial f}{\partial x_{2}}, \ldots, \frac{\partial f}{\partial x_{n}}\right)^{t},
$$

where $t$ is the transposition. The statement of the following lemma is well known for $k=n$ (see [6], [12]).

Lemma 2. Elements $f_{1}, f_{2}, \ldots, f_{k}$ of the algebra $A$ are algebraically dependent if and only if the columns $\partial\left(f_{1}\right), \partial\left(f_{2}\right), \ldots, \partial\left(f_{k}\right)$ are linearly dependent over $A$.

Proof. Let $T\left(y_{1}, y_{2}, \ldots, y_{k}\right) \in F\left[y_{1}, y_{2}, \ldots, y_{k}\right]$ be a nonzero polynomial of minimal degree such that $T\left(f_{1}, f_{2}, \ldots, f_{k}\right)=0$. Applying the derivation $\partial$ to this equality, we get

$$
\partial\left(f_{1}\right) \frac{\partial T}{\partial y_{1}}\left(f_{1}, f_{2}, \ldots, f_{k}\right)+\cdots+\partial\left(f_{k}\right) \frac{\partial T}{\partial y_{k}}\left(f_{1}, f_{2}, \ldots, f_{k}\right)=0 .
$$

This gives a nontrivial linear dependence of the elements $\partial\left(f_{1}\right), \partial\left(f_{2}\right), \ldots, \partial\left(f_{k}\right)$ over $A$.

Now let elements $f_{1}, f_{2}, \ldots, f_{k} \in A$ be algebraically independent. Complete them to an algebraically independent system $f_{1}, \ldots, f_{k}, f_{k+1}, \ldots, f_{n}$ of elements in the quotient field $Q(A)=F\left(x_{1}, x_{2}, \ldots, x_{n}\right)$. Then, by results of [6], [12], we have

$$
\operatorname{det}\left(\partial\left(f_{1}\right), \partial\left(f_{2}\right), \ldots, \partial\left(f_{k}\right), \ldots, \partial\left(f_{n}\right)\right) \neq 0
$$

Therefore, the columns $\partial\left(f_{1}\right), \partial\left(f_{2}\right), \ldots, \partial\left(f_{k}\right)$ are linearly independent over $A$.

Corollary 1. Elements $f_{1}, f_{2}, \ldots, f_{k} \in A$ are algebraically dependent if and only if all the minors of order $k$ of the matrix $\left(\partial\left(f_{1}\right), \partial\left(f_{2}\right), \ldots, \partial\left(f_{k}\right)\right)$ are equal to 0 .

Corollary 2. Elements $f, g \in A$ are algebraically dependent if and only if

$$
\frac{\partial f}{\partial x_{i}} \frac{\partial g}{\partial x_{j}}-\frac{\partial g}{\partial x_{i}} \frac{\partial f}{\partial x_{j}}=0,1 \leq i<j \leq n .
$$

Corollary 3. Elements $f, g \in A$ are algebraically dependent if and only if $[f, g]=0$.

Of course, the Poisson bracket $[f, g]$ can be defined without using free Poisson algebras, just as the vector

$$
\left(\gamma_{1,2}, \gamma_{1,3}, \ldots, \gamma_{1, n}, \gamma_{2,3}, \ldots, \gamma_{n-1, n}\right)
$$


where

$$
\gamma_{i, j}=\frac{\partial f}{\partial x_{i}} \frac{\partial g}{\partial x_{j}}-\frac{\partial g}{\partial x_{i}} \frac{\partial f}{\partial x_{j}}, \quad 1 \leq i<j \leq n .
$$

But our definition of Poisson bracket via free Poisson algebras has certain advantages for working with degrees and highest homogeneous parts. In particular,

$$
\operatorname{deg}[f, g]=\operatorname{deg} f+\operatorname{deg} g
$$

iff $\bar{f}, \bar{g}$ are algebraically independent.

The statement of the following lemma is well known (see [4]).

Lemma 3. If homogeneous polynomials $f, g \in A$ are algebraically dependent, then there exists an element $a \in A$ such that $f=\alpha a^{k}, g=\beta a^{r}$, where $\alpha, \beta \in F, k, r \geq 0$.

Definition 3. For every $f \in A$, the set of elements

$$
\mathcal{C}(f)=\{g \in A \mid[f, g]=0\}
$$

is called a centralizer of $f$ in $A$.

It follows immediately from (1) that $\mathcal{C}(f)$ is a subalgebra of $A$.

The next theorem presents an analogue of the theorem of G. Bergman [3] on centralizers in free associative algebras.

Theorem 1. For every $f \in A \backslash F$, the centralizer $\mathcal{C}(f)$ is a ring of polynomials on a single variable.

Proof. Let $\bar{f}=\alpha a^{k}$, where $\alpha \in F, k \geq 1$, and $a$ is an element that is not a proper power. If $[\bar{f}, \bar{g}] \neq 0$, then $\overline{[f, g]}=[\bar{f}, \bar{g}] \neq 0$. Consequently, if $g \in \mathcal{C}(f)$, then $[\bar{f}, \bar{g}]=0$. Therefore, by Corollary 3 and Lemma 3 , we get $\bar{g}=\beta a^{r}$. Repeating the arguments of Bergman's proof in [3], one can easily show that the algebra $\mathcal{C}(f)$ is finitely generated. Furthermore, by Corollary $3, \mathcal{C}(f)$ has transcendent degree one over $F$. Therefore, the Krull dimension of $\mathcal{C}(f)$ is equal to 1 .

Suppose that $g \in Q(\mathcal{C}(f))$ is integral over $\mathcal{C}(f)$. Since $\mathcal{C}(f) \subseteq A$ and $A$ is integrally closed in $Q(A)$, then $g \in A$. Let

$$
g^{k}+g_{1} g^{k-1}+\cdots+g_{k-1} g+g_{k}=0, g_{i} \in \mathcal{C}(f), 1 \leq i \leq k,
$$

be an integral equation of minimal degree for $g$ over $\mathcal{C}(f)$. Applying the derivation $a d(f)$ to this equation, we get

$$
[g, f]\left(k g^{k-1}+(k-1) g_{1} g^{k-2}+\cdots+g_{k-1}\right)=0 .
$$

Hence $[f, g]=0$, i.e., $g \in \mathcal{C}(f)$ and $\mathcal{C}(f)$ is integrally closed.

Thus, we have proved that $\mathcal{C}(f)$ is a Dedekind domain. Since $F \subset \mathcal{C}(f) \subseteq A$, by a theorem of A. Zaks [13, $\mathcal{C}(f)$ is a ring of polynomials on a single variable.

Problem 1. Is an analogue of Bergman's theorem true for centralizers of free Poisson algebras?

Now we will give two lemmas which will be useful for the calculation of Poisson brackets. Note that calculation of Poisson brackets is strongly related to the Jacobian conjecture (see [2]).

Lemma 4. Let $a \in A \backslash F, c \in C \backslash\{0\}$. Then $[a, c] \neq 0$. 
Proof. It is sufficient to prove the statement of the lemma for homogeneous elements $a, c$. Without loss of generality, we may also assume that the field $F$ is algebraically closed. We will use induction on the number of variables on which $a$ depends. If $a \in F\left[x_{1}\right]$, then the equality $[a, c]=0$ is equivalent to $\left[x_{1}, c\right]=0$. Let us write

$$
c=\sum_{i<j} c_{i j}\left[x_{i}, x_{j}\right], c_{i j} \in A \text {. }
$$

Then we have the equality

$$
\left[c, x_{1}\right]=\sum_{i<j} \sum_{k>1} \frac{\partial c_{i j}}{\partial x_{k}}\left[x_{k}, x_{1}\right]\left[x_{i}, x_{j}\right]+\sum_{i<j} c_{i j}\left[\left[x_{i}, x_{j}\right], x_{1}\right]=0 .
$$

The vector space $P L\left\langle x_{1}, x_{2}, \ldots, x_{n}\right\rangle$ coincides with the space of polynomials on the variables (21). Therefore, the last equality implies

$$
\sum_{i<j} c_{i j}\left[\left[x_{i}, x_{j}\right], x_{1}\right]=0 .
$$

Moreover, since $\left[\left[x_{i}, x_{j}\right], x_{1}\right], 1 \leq i<j \leq n$, are linearly independent elements of the free Lie algebra $L$, we get $c_{i j}=0,1 \leq i<j \leq n$.

Now let $a=f\left(x_{1}, x_{2}, \ldots, x_{n}\right), n>1$, and $\operatorname{deg} a=k$. Consider the linear automorphism $\varphi$ of $A$ such that $\varphi\left(x_{1}\right)=x_{1}, \varphi\left(x_{i}\right)=x_{i}+\alpha_{i} x_{1}, 2 \leq i \leq n$. Then $\varphi(a)=x_{1}^{k} f\left(1, \alpha_{2}, \ldots, \alpha_{n}\right)+g$, where $\operatorname{deg}_{x_{1}}(g)<k$. Choose $\alpha_{2}, \ldots, \alpha_{n}$ such that $f\left(1, \alpha_{2}, \ldots, \alpha_{n}\right)=0$. If this is impossible, then $a=\alpha x_{1}^{k} \in F\left[x_{1}\right]$, and in this case the lemma has already been proved. So, we can assume that $\operatorname{deg}_{x_{1}}(a)=s<k$ and

$$
a=a_{0}+a_{1} x_{1}+\cdots+a_{s} x_{1}^{s},
$$

where $a_{i} \in F\left[x_{2}, \ldots, x_{n}\right], \operatorname{deg}\left(a_{i}\right)=k-i, 0 \leq i \leq s$.

Analogously, the element $c$, as a polynomial in the variables (2), can be represented in the form

$$
c=c_{0}+c_{1} x_{1}+\cdots+c_{r} x_{1}^{r},
$$

where $c_{i} \in C \cap F\left[x_{2}, \ldots, x_{n},\left[x_{1}, x_{2}\right], \ldots\right], 0 \leq i \leq r$. Then

$$
[a, c]=\left[a_{s}, c_{r}\right] x_{1}^{s+r}+h,
$$

where $h$ has degree $<s+r$ on $x_{1}$, as a polynomial on variables (2). Since $a_{s} \in$ $F\left[x_{2}, \ldots, x_{n}\right]$ and $\operatorname{deg}\left(a_{s}\right)=k-s>0$, by the induction assumption we get $\left[a_{s}, c_{r}\right] \neq$ 0 . Consequently, $[a, c] \neq 0$.

Corollary 4. Let $f, g \in A, h \in A \backslash F$. Then

$$
\operatorname{deg}([[f, g], h])=\operatorname{deg}[f, g]+\operatorname{deg} h, \quad \overline{[[f, g], h]}=[\overline{[f, g]}, \bar{h}] .
$$

Lemma 5. Let $f, g, h \in A \backslash F$. Put

$$
m=\operatorname{deg}[f, g]+\operatorname{deg} h, n=\operatorname{deg}[g, h]+\operatorname{deg} f, k=\operatorname{deg}[h, f]+\operatorname{deg} g .
$$

Then $m \leq \max (n, k)$. If $n \neq k$, then $m=\max (n, k)$.

Proof. Since $P L\left\langle x_{1}, x_{2}, \ldots, x_{n}\right\rangle$ is a Lie algebra under the Poisson bracket $[x, y]$, we have

$$
[[f, g], h]+[[g, h], f]+[[h, f], g]=0 .
$$

Now, the simple comparison of the highest parts of the elements $[[f, g], h],[[g, h], f]$, and $[[h, f], g]$, by Corollary 4 , gives the statement of the lemma. 


\section{Subalgebras with a CONDition on Poisson BRACKets}

We fix a $*$-reduced pair of polynomials $f_{1}, f_{2} \in A$ with the condition $\operatorname{deg}\left[f_{1}, f_{2}\right]>$ $\min \left(\operatorname{deg} f_{1}, \operatorname{deg} f_{2}\right)$. For definiteness, we put $m_{1}=\operatorname{deg} f_{1}<\operatorname{deg} f_{2}=m_{2}$, then $\operatorname{deg}\left(\left[f_{1}, f_{2}\right]\right)=m_{1}+m_{0}, m_{0}>0$. By Definition 1 , the elements $\bar{f}_{1}, \bar{f}_{2}$ are algebraically dependent, and $\bar{f}_{2} \notin\left\langle\bar{f}_{1}\right\rangle$. Therefore, $m_{1} \backslash m_{2}$.

This section is devoted to the description of the highest homogeneous parts of elements of the subalgebra $\left\langle f_{1}, f_{2}\right\rangle$. The following algorithm forms the main part of this description.

Algorithm 1. The initial step of algorithm 1 is numbered by 3, for simplicity of notation.

Step 3. Consider the equation

$$
m_{1} s_{1}=m_{2} s_{2}, s_{1}, s_{2} \in Z .
$$

We fix a minimal natural number $s_{2}$ for which this equation has an integer solution, and fix this solution $s_{1}, s_{2}$. Observe that in this solution we have

$$
s_{1}=\frac{m_{2}}{\left(m_{1}, m_{2}\right)}, s_{2}=\frac{m_{1}}{\left(m_{1}, m_{2}\right)} .
$$

Here and in the sequel, $\left(a_{1}, a_{2}, \ldots, a_{n}\right)$ denotes the greatest common divisor of the elements $a_{1}, a_{2}, \ldots, a_{n}$. Since $m_{1} \backslash m_{2}$, we have $s_{2}>1$.

Lemma 6. The elements of the type

$$
f_{1}^{i_{1}} f_{2}^{i_{2}}, i_{2}<s_{2},
$$

have different degrees for different values of $i_{1}, i_{2}$.

Proof. Keeping in mind the equality

$$
\operatorname{deg}\left(f_{1}^{i_{1}} f_{2}^{i_{2}}\right)=m_{1} i_{1}+m_{2} i_{2},
$$

suppose that

$$
m_{1} i_{1}+m_{2} i_{2}=m_{1} j_{1}+m_{2} j_{2}, i_{2}, j_{2}<s_{2} .
$$

Without loss of generality, we can assume that $i_{2} \geq j_{2}$. Then

$$
m_{1}\left(j_{1}-i_{1}\right)=m_{2}\left(i_{2}-j_{2}\right), 0 \leq i_{2}-j_{2}<s_{2} .
$$

By the condition on choosing $s_{2}$, we have $i_{2}=j_{2}$, and, consequently, $i_{1}=j_{1}$.

Since $\operatorname{deg}\left(f_{1}^{s_{1}}\right)=m_{1} s_{1}=m_{2} s_{2}=\operatorname{deg}\left(f_{2}^{s_{2}}\right)$ and $\bar{f}_{1}, \bar{f}_{2}$ are algebraically dependent, by Lemma 3 we may assume that $\bar{f}_{1}^{s_{1}}=\bar{f}_{2}{ }^{s_{2}}$. Hence the element $f=f_{2}^{s_{2}}-f_{1}^{s_{1}}$ has degree less than $m_{2} s_{2}$.

Assume that $\bar{f} \in\left\langle\bar{f}_{1}, \bar{f}_{2}\right\rangle$. Since $\bar{f}_{1}, \bar{f}_{2}$ are algebraically dependent, by Lemma 3 there exists an element $a \in A$ such that $\bar{f}_{1}=\alpha a^{k}, \bar{f}_{2}=\beta a^{s}$ for some $\alpha, \beta \in F$. Therefore, the space of elements of type $\bar{f}_{1}{ }^{i_{1}} \bar{f}_{2}{ }^{i_{2}}$ of fixed degree is one dimensional. Hence $\bar{f}=\alpha_{i_{1}, i_{2}} \bar{f}_{1}{ }^{i_{1}} \bar{f}_{2}{ }^{i_{2}}$. Since $m_{1} i_{1}+m_{2} i_{2}=\operatorname{deg} f<m_{2} s_{2}$, the element $f_{1}{ }^{i_{1}} f_{2}{ }^{{ }^{2}}$ is of the type given in Lemma 6 . We replace $f$ by $f-\alpha_{i_{1}, i_{2}} f_{1}^{i_{1}} f_{2}^{i_{2}}$ and note that $\operatorname{deg}\left(f-\alpha_{i_{1}, i_{2}} f_{1}^{i_{1}} f_{2}^{i_{2}}\right)<\operatorname{deg} f$. After several such reductions, we get an element

$$
f_{3}=f_{2}^{s_{2}}-f_{1}^{s_{1}}-\sum_{i_{1}, i_{2}} \alpha_{i_{1}, i_{2}} f_{1}^{i_{1}} f_{2}^{i_{2}},
$$

where $m_{1} i_{1}+m_{2} i_{2}<m_{2} s_{2}$ and $\bar{f}_{3} \notin\left\langle\bar{f}_{1}, \bar{f}_{2}\right\rangle$. Observe that $f_{3} \neq 0$ since $f_{1}, f_{2}$ are algebraically independent. We put $m_{3}=\operatorname{deg} f_{3}$. 
Lemma 7. $\overline{\left[f_{1}, f_{3}\right]}=s_{2} \overline{\left[f_{1}, f_{2}\right]} \bar{f}_{2}{ }^{s_{2}-1}$.

Proof. A straightforward calculation gives

$$
\left[f_{1}, f_{3}\right]=\left[f_{1}, f_{2}\right]\left(s_{2} f_{2}^{s_{2}-1}-\sum_{i_{1}, i_{2}} i_{2} \alpha_{i_{1}, i_{2}} f_{1}^{i_{1}} f_{2}^{i_{2}-1}\right) .
$$

Since $m_{1} i_{1}+m_{2} i_{2}<m_{2} s_{2}$, the inequality

$$
\operatorname{deg}\left(f_{1}^{i_{1}} f_{2}^{i_{2}-1}\right)=m_{1} i_{1}+m_{2}\left(i_{2}-1\right)<m_{2} s_{2}-m_{2}=\operatorname{deg}\left(f_{2}^{s_{2}-1}\right)
$$

completes the proof.

Corollary 5. $m_{0}+m_{2}\left(s_{2}-1\right) \leq m_{3}<m_{2} s_{2}$.

Proof. By Lemma 7 we get

$$
m_{1}+m_{3} \geq \operatorname{deg}\left[f_{1}, f_{3}\right]=m_{1}+m_{0}+m_{2}\left(s_{2}-1\right) ;
$$

hence $m_{3} \geq m_{0}+m_{2}\left(s_{2}-1\right)$. The inequality $m_{3}<m_{2} s_{2}$ follows from the definition of $f_{3}$.

Step 3 of algorithm 1 completes its work by testing whether the elements $\bar{f}_{1}, \bar{f}_{3}$ are algebraically dependent. If they are algebraically independent, algorithm 1 finishes its work too.

Suppose now that, after $t \geq 3$ steps, algorithm 1 produces a set of polynomials

$$
f_{1}, f_{2}, \ldots, f_{t}, \operatorname{deg} f_{i}=m_{i}, 1 \leq i \leq t,
$$

which satisfy the following conditions:

C1) Let $s_{i}, 2 \leq i \leq t-1$, be a minimal natural number for which the equation

$$
m_{1} r_{1}+m_{2} r_{2}+\cdots+m_{i-1} r_{i-1}=m_{i} s_{i}
$$

has an integer solution $r_{1}, r_{2}, \ldots, r_{i-1}$. Then this equation has a solution satisfying the inequalities $0<r_{1}, 0 \leq r_{2}<s_{2}, \ldots, 0 \leq r_{i-1}<s_{i-1}$.

C2) The elements $\bar{f}_{1}, \bar{f}_{2}, \ldots, \bar{f}_{t-1}$ are mutually algebraically dependent, and, for every $j, 2 \leq j \leq t, \bar{f}_{j} \notin\left\langle\bar{f}_{1}, \bar{f}_{2}, \ldots, \bar{f}_{j-1}\right\rangle$ holds.

C3) $\overline{\left[f_{1}, f_{i}\right]}=s_{2} \ldots s_{i-1} \overline{\left[f_{1}, f_{2}\right]} \bar{f}_{2}{ }^{s_{2}-1} \ldots \bar{f}_{i-1}^{s_{i-1}-1}$, for every $i, 3 \leq i \leq t$.

C4) $m_{0}+m_{2}\left(s_{2}-1\right)+\cdots+m_{i-1}\left(s_{i-1}-1\right) \leq m_{i}<m_{i-1} s_{i-1}$, for all $i, 3 \leq i \leq t$, and $\left(m_{1}, m_{2}\right)>\left(m_{1}, m_{2}, m_{3}\right)>\cdots>\left(m_{1}, m_{2}, \ldots, m_{t-1}\right)$.

If $\bar{f}_{1}, \bar{f}_{t}$ are algebraically independent, then algorithm 1 finishes its work. Otherwise, the next step starts.

Step $t+1(t \geq 3)$. Denote by $s_{t}$ a minimal natural number for which the equation

$$
m_{1} r_{1}+m_{2} r_{2}+\cdots+m_{t-1} r_{t-1}=m_{t} s_{t}
$$

has an integer solution $r_{1}, r_{2}, \ldots, r_{t-1}$.

Lemma 8. Equation (5) has an integer solution $r_{1}, r_{2}, \ldots, r_{t-1}$, satisfying the inequalities $0<r_{1}, 0 \leq r_{2}<s_{2}, \ldots, 0 \leq r_{t-1}<s_{t-1}$.

Proof. Applying successively condition C1), we can substitute $r_{i}$ in equation (5) by their remainders modulo $s_{i}$, for every $i=t-1, \ldots, 2$. In this way, we find a solution of (5) satisfying the inequalities $0 \leq r_{i}<s_{i}, 2 \leq i \leq t-1$. By C4), we have

$$
m_{t} \geq m_{0}+m_{2}\left(s_{2}-1\right)+\cdots+m_{t-1}\left(s_{t-1}-1\right) .
$$


Therefore,

$$
\begin{aligned}
m_{1} r_{1}+m_{2} r_{2}+\cdots+m_{t-1} r_{t-1}=m_{t} s_{t} & \geq m_{t} \\
& \geq m_{0}+m_{2}\left(s_{2}-1\right)+\cdots+m_{t-1}\left(s_{t-1}-1\right) .
\end{aligned}
$$

Since

$$
m_{2} r_{2}+\cdots+m_{t-1} r_{t-1} \leq m_{2}\left(s_{2}-1\right)+\cdots+m_{t-1}\left(s_{t-1}-1\right),
$$

we get $m_{1} r_{1} \geq m_{0}>0$, i.e., $r_{1}>0$.

By Lemma 3 and Lemma $8, \bar{f}_{t} \in\left\langle\bar{f}_{1}, \ldots, \bar{f}_{t-1}\right\rangle$ if and only if $s_{t}=1$. Now condition C2) gives $s_{t}>1$. Therefore, $\left(m_{1}, m_{2}, \ldots, m_{t-1}\right)>\left(m_{1}, m_{2}, \ldots, m_{t}\right)$, since otherwise $\left(m_{1}, m_{2}, \ldots, m_{t-1}\right) \mid m_{t}$ and equation (5) would admit a solution with $s_{t}=1$.

Lemma 9. The elements of the type

$$
f_{1}^{i_{1}} f_{2}^{i_{2}} \ldots f_{t}^{i_{t}}, i_{2}<s_{2}, \ldots, i_{t}<s_{t}
$$

have different degrees for different values of $i_{1}, i_{2}, \ldots, i_{t}$.

Proof. Consider the equation

$$
m_{1} i_{1}+m_{2} i_{2}+\cdots+m_{t} i_{t}=m_{1} j_{1}+m_{2} j_{2}+\cdots+m_{t} j_{t}
$$

where $i_{r}, j_{r}<s_{r}, 2 \leq r \leq t$. By definition of $s_{t}$, we get $i_{t}=j_{t}$, which yields

$$
m_{1} i_{1}+m_{2} i_{2}+\cdots+m_{t-1} i_{t-1}=m_{1} j_{1}+m_{2} j_{2}+\cdots+m_{t-1} j_{t-1} .
$$

Applying successively condition C1), we get $i_{t-1}=j_{t-1}, \ldots, i_{1}=j_{1}$.

In the sequel, we fix a solution $r_{1}, r_{2}, \ldots, r_{t-1}$ of equation (5) satisfying the conditions of Lemma 8. Then, by Lemma 3,

$$
\bar{f}_{t}^{s_{t}}=\alpha_{r_{1}, \ldots, r_{t-1}} \bar{f}_{1}^{r_{1}} \bar{f}_{2}^{r_{2}} \ldots \bar{f}_{t-1}^{r_{t-1}} \text {. }
$$

Consider the element

$$
f=f_{t}^{s_{t}}-\alpha_{r_{1}, \ldots, r_{t-1}} f_{1}^{r_{1}} f_{2}^{r_{2}} \ldots f_{t-1}^{r_{t-1}}
$$

We have $\operatorname{deg} f<m_{t} s_{t}$. Assume that $\bar{f} \in\left\langle\bar{f}_{1}, \bar{f}_{2}, \ldots, \bar{f}_{t}\right\rangle$. Since the elements $\bar{f}_{1}, \bar{f}_{2}, \ldots, \bar{f}_{t}$ are mutually algebraically dependent, the space spanned by the elements of the type $\bar{f}_{1}^{i_{1}} \bar{f}_{2}{ }^{i_{2}} \ldots \bar{f}_{t} i_{t}$ with a fixed degree is one dimensional. Therefore,

$$
\bar{f}=\alpha_{i_{1}, i_{2}, \ldots, i_{t}} \bar{f}_{1}^{i_{1}} \bar{f}_{2}^{i_{2}} \ldots \bar{f}_{t}^{i_{t}}
$$

for some $i_{1}, i_{2}, \ldots, i_{t}$ such that $m_{1} i_{1}+m_{2} i_{2}+\cdots+m_{t} i_{t}=\operatorname{deg} f$. Arguing as in the proof of Lemma 8 , by Lemma 8 and condition $\mathrm{C} 1$ ), we may assume that $i_{2}<s_{2}, \ldots, i_{t}<s_{t}$. Thus the element $f_{1}^{i_{1}} f_{2}^{i_{2}} \ldots f_{t}^{i_{t}}$ has a form given in Lemma 9 , and we replace $f$ by the element $f-\alpha_{i_{1}, i_{2}, \ldots, i_{t}} f_{1}^{i_{1}} f_{2}^{i_{2}} \ldots f_{t}^{i_{t}}$. Repeating, if necessary, such reductions, eventually we get an element

$$
f_{t+1}=f_{t}^{s_{t}}-\alpha_{r_{1}, \ldots, r_{t-1}} f_{1}^{r_{1}} f_{2}^{r_{2}} \ldots f_{t-1}^{r_{t-1}}-\sum_{i_{1}, i_{2}, \ldots, i_{t}} \alpha_{i_{1}, i_{2}, \ldots, i_{t}} f_{1}^{i_{1}} f_{2}^{i_{2}} \ldots f_{t}^{i_{t}},
$$

where $m_{1} r_{1}+\cdots+m_{t-1} r_{t-1}=m_{t} s_{t}, m_{1} i_{1}+\cdots+m_{t} i_{t}<m_{t} s_{t}$, and $\bar{f}_{t+1} \notin$ $\left\langle\bar{f}_{1}, \ldots, \bar{f}_{t}\right\rangle$ or $f_{t+1}=0$. The next lemma shows, in particular, that $f_{t+1} \neq 0$. We put $m_{t+1}=\operatorname{deg} f_{t+1}$.

Lemma 10. $\overline{\left[f_{1}, f_{t+1}\right]}=s_{2} \ldots s_{t} \overline{\left[f_{1}, f_{2}\right]} \bar{f}_{2}{ }^{s_{2}-1} \ldots \bar{f}_{t} s_{t}-1$. 
Proof. By (1) and ([6),

$$
\begin{aligned}
{\left[f_{1}, f_{t+1}\right]=} & s_{t}\left[f_{1}, f_{t}\right] f_{t}^{s_{t}-1}-\alpha_{r_{1}, \ldots, r_{t-1}}\left(\sum_{j \geq 2} r_{j}\left[f_{1}, f_{j}\right] f_{1}^{r_{1}} \ldots f_{j}^{r_{j}-1} \ldots f_{t-1}^{r_{t-1}}\right) \\
& -\sum_{i_{1}, i_{2}, \ldots, i_{t}} \alpha_{i_{1}, i_{2}, \ldots, i_{t}}\left(\sum_{j \geq 2} i_{j}\left[f_{1}, f_{j}\right] f_{1}^{i_{1}} \ldots f_{j}^{i_{j}-1} \ldots f_{t}^{i_{t}}\right) .
\end{aligned}
$$

By condition C3),

$$
\operatorname{deg}\left(\left[f_{1}, f_{t}\right] f_{t}^{s_{t}-1}\right)=m_{1}+m_{0}+m_{2}\left(s_{2}-1\right)+\cdots+m_{t}\left(s_{t}-1\right)=d .
$$

Furthermore, equality (5) gives

$$
\begin{aligned}
\operatorname{deg} & \left(\left[f_{1}, f_{j}\right] f_{1}^{r_{1}} \ldots f_{j}^{r_{j}-1} \ldots f_{t-1}^{r_{t-1}}\right)=m_{1}+m_{0}+m_{2}\left(s_{2}-1\right)+\cdots+m_{j-1}\left(s_{j-1}-1\right) \\
& +m_{1} r_{1}+\cdots+m_{j}\left(r_{j}-1\right)+\cdots+m_{t-1} r_{t-1} \\
= & m_{1}+m_{0}+m_{2}\left(s_{2}-1\right)+\cdots+m_{j-1}\left(s_{j-1}-1\right)+m_{t} s_{t}-m_{j} \\
= & d+m_{t}-m_{j}-m_{j}\left(s_{j}-1\right)-\cdots-m_{t-1}\left(s_{t-1}-1\right) .
\end{aligned}
$$

By C4), for every $j<t$,

$$
\begin{aligned}
& m_{j}+m_{j}\left(s_{j}-1\right)+\cdots+m_{t-1}\left(s_{t-1}-1\right)=m_{j} s_{j}+\cdots+m_{t-1}\left(s_{t-1}-1\right) \\
& \quad>m_{j+1}+m_{j+1}\left(s_{j+1}-1\right)+\cdots+m_{t-1}\left(s_{t-1}-1\right) \\
& >\cdots \\
& >m_{t-1}+m_{t-1}\left(s_{t-1}-1\right)=m_{t-1} s_{t-1}>m_{t} .
\end{aligned}
$$

Hence

$$
\operatorname{deg}\left(\left[f_{1}, f_{j}\right] f_{1}^{r_{1}} \ldots f_{j}^{r_{j}-1} \ldots f_{t-1}^{r_{t-1}}\right)<d .
$$

Analogously, the condition $m_{1} i_{1}+\cdots+m_{t} i_{t}<m_{t} s_{t}$ gives

$$
\operatorname{deg}\left(\left[f_{1}, f_{j}\right] f_{1}^{i_{1}} \ldots f_{j}^{i_{j}-1} \ldots f_{t}^{i_{t}}\right)<d .
$$

Now, by condition C3),

$$
\overline{\left[f_{1}, f_{t+1}\right]}=s_{t} \overline{\left[f_{1}, f_{t}\right]} \bar{f}_{t}{ }^{s_{t}-1}=s_{2} \ldots s_{t} \overline{\left[f_{1}, f_{2}\right]} \bar{f}_{2}{ }^{s_{2}-1} \ldots \bar{f}_{t}{ }^{s_{t}-1},
$$

which proves the lemma.

Corollary 6. $m_{0}+m_{2}\left(s_{2}-1\right)+\cdots+m_{t}\left(s_{t}-1\right) \leq m_{t+1}<m_{t} s_{t}$.

Thus, algorithm 1 is described and justified. It will necessarily stop after a finite number of steps because of the strict decrease of the sequence of greatest common divisors in $\mathrm{C} 4$ ).

In the sequel, we suppose that algorithm 1 has finished its work and produced a set of elements (4) which satisfies conditions $\mathrm{C} 1)-\mathrm{C} 4$ ) and the condition

C5) $\bar{f}_{1}, \bar{f}_{t}$ are algebraically independent.

Lemma 11. The highest homogeneous parts of the elements of the type

$$
f_{1}^{i_{1}} f_{2}^{i_{2}} \ldots f_{t}^{i_{t}}, i_{2}<s_{2}, \ldots, i_{t-1}<s_{t-1},
$$

are linearly independent. 
Proof. As in Lemma 9, it is easy to see that the elements of the type

$$
f_{1}^{i_{1}} f_{2}^{i_{2}} \ldots f_{t-1}^{i_{t-1}}, i_{2}<s_{2}, \ldots, i_{t-1}<s_{t-1},
$$

have different degrees for different values of $i_{1}, i_{2}, \ldots, i_{t-1}$.

Since the elements $\bar{f}_{1}, \bar{f}_{2}, \ldots, \bar{f}_{t-1}$ are mutually algebraically dependent, by Lemma 3 there exists an element $a \in A$ such that $\bar{f}_{i}=\alpha_{i} a^{k_{i}}, 1 \leq i \leq t-1$. The linear dependence of the highest parts of the elements of type (7) would give the nontrivial equality of the form

$$
F_{0}(a)+F_{1}(a) \bar{f}_{t}+\cdots+F_{s}(a) \bar{f}_{t}^{s}=0 .
$$

This contradicts condition C5).

Consider now the set of commutative words

$$
f_{1}^{i_{1}} f_{2}^{i_{2}} \ldots f_{t}^{i_{t}}
$$

on alphabet (4), without the restrictions imposed in (7). Define a degree function $d$ on these words, by setting

$$
d\left(f_{1}^{i_{1}} f_{2}^{i_{2}} \ldots f_{t}^{i_{t}}\right)=\operatorname{deg}\left(f_{2}^{i_{2}} \ldots f_{t}^{i_{t}}\right)=m_{2} i_{2}+\cdots+m_{t} i_{t} .
$$

By their construction in the process of algorithm 1 (see (6) ), the elements $f_{k+1}$ satisfy the following relations:

$$
f_{k}^{s_{k}}=f_{k+1}+\alpha_{r_{1}, \ldots, r_{k-1}} f_{1}^{r_{1}} f_{2}^{r_{2}} \ldots f_{k-1}^{r_{k-1}}+\sum_{i_{1}, i_{2}, \ldots, i_{k}} \alpha_{i_{1}, i_{2}, \ldots, i_{k}} f_{1}^{i_{1}} f_{2}^{i_{2}} \ldots f_{k}^{i_{k}}
$$

where $m_{1} r_{1}+\cdots+m_{k-1} r_{k-1}=m_{k} s_{k}, m_{1} i_{1}+\cdots+m_{k} i_{k}<m_{k} s_{k}, r_{j}, i_{j}<s_{j}$ for $2 \leq j \leq k-1$, and $i_{k}<s_{k}, 2 \leq k \leq t-1$.

Lemma 12. The set of words of type (7) forms a basis of the algebra $\left\langle f_{1}, f_{2}\right\rangle$.

Proof. We prove first that the words (8) can be expressed as linear combinations of words of type (7). The process of reduction consists of substituting the elements $f_{k}^{s_{k}}, 2 \leq k \leq t-1$, by the right parts of the equalities (9). It suffices to prove that the $d$-degree of the right part of (9) is less than $d\left(f_{k}^{s_{k}}\right)$; then our statement will easily follow by induction on the $d$-degree. By $\mathrm{C} 4$ ), we have

$$
\begin{aligned}
d\left(f_{k}^{s_{k}}\right) & =m_{k} s_{k}>m_{k+1}=d\left(f_{k+1}\right), \\
d\left(f_{k+1}\right) & \geq m_{0}+m_{2}\left(s_{2}-1\right)+\cdots+m_{k}\left(s_{k}-1\right) .
\end{aligned}
$$

Since $r_{j}, i_{j}<s_{j}$ for $2 \leq j \leq k-1$, and $i_{k}<s_{k}$, we get

$$
\begin{aligned}
d\left(f_{1}^{r_{1}} f_{2}^{r_{2}} \ldots f_{k-1}^{r_{k-1}}\right) & =m_{2} r_{2}+\cdots+m_{k-1} r_{k-1}<d\left(f_{k+1}\right), \\
d\left(f_{1}^{i_{1}} f_{2}^{i_{2}} \ldots f_{k}^{i_{k}}\right) & =m_{2} i_{2}+\cdots+m_{k} i_{k}<d\left(f_{k+1}\right) .
\end{aligned}
$$

Thus, the linear subspace spanned by the words of type (7) forms a subalgebra which obviously coincides with $\left\langle f_{1}, f_{2}\right\rangle$. Now, Lemma 11 completes the proof.

Define a linear order $\leq$ on the set of words of type (7), which corresponds to the inverse lexicographic order on the set of $t$-tuples $\left(i_{1}, i_{2}, \ldots, i_{t}\right)$. For an element $f \in\left\langle f_{1}, f_{2}\right\rangle$, we will denote by $\{f\}$ the leading term of $f$ with respect to $\leq$ (with coefficient 1). For a word $u=f_{1}^{i_{1}} f_{2}^{i_{2}} \ldots f_{t}^{i_{t}}$, we put also $[u]=f_{2}^{i_{2}} \ldots f_{t}^{i_{t}}$.

Lemma 13. Let $u, v$ be words of type (7) and $u=f_{1}^{i_{1}}[u], v=f_{1}^{j_{1}}[v]$. Then $u \leq v$ iff $d(u)<d(v)$ or $d(u)=d(v), i_{1} \leq j_{1}$. Moreover, $d(u)=d(v)$ iff $[u]=[v]$. 
Proof. By definition, we have $u \leq v$ iff $[u]<[v]$ or $[u]=[v], i_{1} \leq j_{1}$. Therefore, it is sufficient to prove that if $[u]<[v]$, then $d(u)<d(v)$.

Take $[u]=f_{2}^{i_{2}} \ldots f_{t}^{i_{t}},[v]=f_{2}^{j_{2}} \ldots f_{t}^{j_{t}}$, and assume that $i_{t}=j_{t}, \ldots, i_{k+1}=$ $j_{k+1}, i_{k}<j_{k}$. Then we obtain, by C4),

$$
\begin{aligned}
d(u) & =m_{2} i_{2}+\cdots+m_{k} i_{k}+m_{k+1} i_{k+1}+\cdots+m_{t} i_{t} \\
& \leq m_{2}\left(s_{2}-1\right)+\cdots+m_{k-1}\left(s_{k-1}-1\right)+m_{k} i_{k}+m_{k+1} i_{k+1}+\cdots+m_{t} i_{t} \\
& <m_{k}+m_{k} i_{k}+m_{k+1} i_{k+1}+\cdots+m_{t} i_{t} \\
& \leq m_{k} j_{k}+m_{k+1} j_{k+1}+\cdots+m_{t} j_{t} \leq d(v) .
\end{aligned}
$$

This lemma and the proof of Lemma 12 imply

Corollary 7. The leading term of the right part of equality (99) is equal to $f_{k+1}$.

Lemma 14. Let $u, v, w$ be arbitrary words of type (7). If $u<v$, then $\{u w\}<\{v w\}$.

Proof. We will prove the lemma by induction on $d(v)+d(w)$. A base for the induction is given by the evident case $d(v)+d(w)=0$, when $u, v, w$ depend only on $f_{1}$. Assume now that for arbitrary words $u_{1}, v_{1}, w_{1}$ of type (7), with the condition $u_{1}<v_{1}, d\left(v_{1}\right)+d\left(w_{1}\right)<d(v)+d(w)$, the inequality $\left\{u_{1} w_{1}\right\}<\left\{v_{1} w_{1}\right\}$ is true. With this assumption, it is easy to see that for arbitrary elements $f, g \in\left\langle f_{1}, f_{2}\right\rangle$, such that $d(f)+d(g)<d(v)+d(w)$, the equality

$$
\{f g\}=\{\{f\}\{g\}\}
$$

holds.

First, we consider the case $w=f_{r}, 1 \leq r \leq t$. Note that the statement of the lemma is trivial for $w=f_{1}$. Moreover, without loss of generality, we can take $u=[u], v=[v]$. Now we proceed with the reverse induction on $r$. If $w=f_{t}$, then the statement of the lemma is trivial too. Suppose that it is true for $w=f_{l}, l>r$.

Denote by $k$ the maximal natural number such that $v$ depends on $f_{k}$. Since $u<v$, then $u$ also does not depend on $f_{k+1}, \ldots, f_{t}$. Consequently, if $r>k$, we have

$$
\left\{u f_{r}\right\}=u f_{r}<v f_{r}=\left\{v f_{r}\right\} .
$$

If $r=k$, then we have $u=u_{1} f_{r}^{i_{r}}, v=v_{1} f_{r}^{j_{r}}$, where $u_{1}, v_{1}$ do not depend on $f_{r}$ and $i_{r} \leq j_{r}$. If $j_{r}<s_{r}-1$, then

$$
\left\{u f_{r}\right\}=u_{1} f_{r}^{i_{r}+1}<v_{1} f_{r}^{j_{r}+1}=\left\{v f_{r}\right\} .
$$

If $j_{r}=s_{r}-1$, then the subword $f_{r}^{s_{r}}$ of the word $v f_{r}=v_{1} f_{r}^{s_{r}}$ is replaced by the right part of equality (9), with $k=r$. By Corollary 7 and equality (10), we obtain

$$
\left\{v f_{r}\right\}=v_{1} f_{r+1} \text {. }
$$

In case $i_{r}=s_{r}-1$ we have $u_{1}<v_{1}$, and analogously

$$
\left\{u f_{r}\right\}=u_{1} f_{r+1}<v_{1} f_{r+1} .
$$

If $i_{r}<s_{r}-1$, then by condition $\left.\mathrm{C} 4\right)$ we get

$$
d\left(u f_{r}\right) \leq m_{2}\left(s_{2}-1\right)+\cdots+m_{r}\left(s_{r}-1\right)<m_{r+1} \leq d\left(v_{1} f_{r+1}\right) .
$$

Now consider the case $r<k$. If $u$ also depends on $f_{k}$, then we have $u=u_{1} f_{k}$, $v=v_{1} f_{k}, u_{1}<v_{1}$. A composition of two inductions gives

$$
\left\{u f_{r}\right\}=\left\{\left(u_{1} f_{r}\right) f_{k}\right\}=\left\{\left\{u_{1} f_{r}\right\} f_{k}\right\}<\left\{\left\{v_{1} f_{r}\right\} f_{k}\right\}=\left\{\left(v_{1} f_{r}\right) f_{k}\right\}=\left\{v f_{r}\right\} .
$$


If $u$ does not depend on $f_{k}$, then $v=v_{1} f_{k}$ and by condition C4) we have $d(u)<m_{k}$. Since $f_{k} \leq v$, the previous case gives $\left\{f_{k} f_{r}\right\} \leq\left\{v f_{r}\right\}$, i.e., $f_{k} f_{r} \leq\left\{v f_{r}\right\}$. Note that

$$
d\left(u f_{r}\right)<m_{k}+m_{r}=d\left(f_{r} f_{k}\right) .
$$

Then

$$
\left\{u f_{r}\right\}<f_{r} f_{k} \leq\left\{v f_{r}\right\} .
$$

We now turn to the case $w=w_{1} w_{2}$, where $w_{1}$ and $w_{2}$ are nontrivial words of type (7). The induction on the length of $w$ relatively to the variables (4) gives

$$
\{u w\}=\left\{\left(u w_{1}\right) w_{2}\right\}=\left\{\left\{u w_{1}\right\} w_{2}\right\}<\left\{\left\{v w_{1}\right\} w_{2}\right\}=\{v w\} .
$$

Corollary 8. Let $f, g \in\left\langle f_{1}, f_{2}\right\rangle$ be arbitrary elements. Then

$$
\{f g\}=\{\{f\}\{g\}\} \text {. }
$$

Lemma 15. $m_{i} \geq\left(m_{0}+m_{2}\left(s_{2}-1\right)\right) s_{3} \ldots s_{i-1}, 3 \leq i \leq t$.

Proof. We prove the statement of the lemma by induction on $i$. If $i=3$, it follows from condition C4). Assume that it is true for every $r<i$. By C4),

$$
m_{i} \geq\left(m_{0}+m_{2}\left(s_{2}-1\right)\right)+m_{3}\left(s_{3}-1\right)+\cdots+m_{i-1}\left(s_{i-1}-1\right) .
$$

Therefore, by the induction assumption,

$$
\begin{aligned}
m_{i} & \geq\left(m_{0}+m_{2}\left(s_{2}-1\right)\right)\left(1+\left(s_{3}-1\right)+s_{3}\left(s_{4}-1\right)+\cdots+s_{3} \ldots s_{i-2}\left(s_{i-1}-1\right)\right) \\
& =\left(m_{0}+m_{2}\left(s_{2}-1\right)\right) s_{3} \ldots s_{i-1} .
\end{aligned}
$$

For every natural number $k$, we define the numbers $r_{2}, r_{3}, \ldots, r_{t-1}, q_{t}$, by means of the following equalities:

$$
\begin{aligned}
k= & s_{2} q_{3}+r_{2}, 0 \leq r_{2}<s_{2}, \\
q_{3}= & s_{3} q_{4}+r_{3}, 0 \leq r_{3}<s_{3}, \\
& \cdots \\
q_{t-1}= & s_{t-1} q_{t}+r_{t-1}, 0 \leq r_{t-1}<s_{t-1} .
\end{aligned}
$$

Lemma 16. $\left\{f_{2}^{k}\right\}=f_{2}^{r_{2}} f_{3}^{r_{3}} \ldots f_{t-1}^{r_{t-1}} f_{t}^{q_{t}}$.

Proof. We will show, by reverse induction on $i$, that

$$
\left\{f_{i}^{q_{i}}\right\}=f_{i}^{r_{i}} \ldots f_{t-1}^{r_{t-1}} f_{t}^{q_{t}}
$$

for every $i=2, \ldots, t$, with $q_{2}=k$. The base of the induction, for $i=t$, is evident. By Corollary 8, we have

$$
\left\{f_{i-1}^{q_{i-1}}\right\}=\left\{\left(f_{i-1}^{s_{i-1}}\right)^{q_{i}} f_{i-1}^{r_{i-1}}\right\}=\left\{\left\{\left\{f_{i-1}^{s_{i-1}}\right\}^{q_{i}}\right\} f_{i-1}^{r_{i-1}}\right\} .
$$

Observe that, by Corollary $7,\left\{f_{i-1}^{s_{i-1}}\right\}=f_{i}$. Hence, by the induction assumption,

$$
\left\{f_{i-1}^{q_{i-1}}\right\}=\left\{\left\{f_{i}^{q_{i}}\right\} f_{i-1}^{r_{i-1}}\right\}=\left\{f_{i-1}^{r_{i-1}} f_{i}^{r_{i}} \ldots f_{t-1}^{r_{t-1}} f_{t}^{q_{t}}\right\}=f_{i-1}^{r_{i-1}} f_{i}^{r_{i}} \ldots f_{t-1}^{r_{t-1}} f_{t}^{q_{t}},
$$

which proves the lemma.

Corollary 9. $\left\{f_{1}^{r} f_{2}^{k}\right\}=\left\{f_{1}^{r_{1}} f_{2}^{k_{1}}\right\}$ iff $r_{1}=r, k_{1}=k$. 
Theorem 2. Let $G(x, y) \in F[x, y]$ and $\operatorname{deg}_{y}(G(x, y))=k$. Then

$$
\operatorname{deg}\left(G\left(f_{1}, f_{2}\right)\right) \geq m_{2} k+\left(m_{0}-m_{2}\right)\left[\frac{k \cdot\left(m_{1}, m_{2}\right)}{m_{1}}\right],
$$

where $[\alpha]$ is the integer part of $\alpha$.

Proof. By Corollary 9 and Lemma 11, we have

$$
\operatorname{deg}\left(G\left(f_{1}, f_{2}\right)\right) \geq \operatorname{deg}\left(\left\{f_{2}^{k}\right\}\right) .
$$

Return to equalities (11). By Lemma 16,

$$
\operatorname{deg}\left(\left\{f_{2}^{k}\right\}\right)=m_{2} r_{2}+\cdots+m_{t-1} r_{t-1}+m_{t} q_{t} .
$$

Now, Lemma 15 gives

$\operatorname{deg}\left(G\left(f_{1}, f_{2}\right)\right) \geq m_{2} r_{2}+\left(m_{0}+m_{2}\left(s_{2}-1\right)\right)\left(r_{3}+s_{3} r_{4}+s_{3} s_{4} r_{5}+\cdots+s_{3} \ldots s_{t-1} q_{t}\right)$.

By (11),

$$
r_{3}+s_{3}\left(r_{4}+s_{4}\left(r_{5}+\cdots+s_{t-2}\left(r_{t-1}+s_{t-1} q_{t}\right) \ldots\right)\right)=q_{3} .
$$

Consequently,

$$
\begin{aligned}
\operatorname{deg}\left(G\left(f_{1}, f_{2}\right)\right) & \geq m_{2} r_{2}+\left(m_{0}+m_{2}\left(s_{2}-1\right)\right) q_{3} \\
& =m_{2}\left(r_{2}+s_{2} q_{3}\right)+\left(m_{0}-m_{2}\right) q_{3}=m_{2} k+\left(m_{0}-m_{2}\right) q_{3} .
\end{aligned}
$$

Since $q_{3}=\left[\frac{k}{s_{2}}\right]$, by equality (3) we have $q_{3}=\left[\frac{k \cdot\left(m_{1}, m_{2}\right)}{m_{1}}\right]$. This proves the theorem.

\section{The estimation of Degrees in the General CASE}

The main result of the paper is the following theorem.

Theorem 3. Let $g_{1}, g_{2}$ be an arbitrary $*$-reduced pair of polynomials over a field $F$ of characteristic 0 , and let $n=\operatorname{deg} g_{1}<m=\operatorname{deg} g_{2}, p=\frac{n}{(n, m)}, s=\frac{m}{(n, m)}, N=$ $N\left(g_{1}, g_{2}\right)=\frac{m n}{(n, m)}-m-n+\operatorname{deg}\left[g_{1}, g_{2}\right]$. Suppose that $G(x, y) \in F[x, y]$. If $\operatorname{deg}_{y}(G(x, y))=p q+r$, where $0 \leq r<p$, then

$$
\operatorname{deg}\left(G\left(g_{1}, g_{2}\right)\right) \geq q N+m r .
$$

If $\operatorname{deg}_{x}(G(x, y))=s q_{1}+r_{1}$, where $0 \leq r_{1}<s$, then

$$
\operatorname{deg}\left(G\left(g_{1}, g_{2}\right)\right) \geq q_{1} N+n r_{1} .
$$

Proof. Put $f_{1}=g_{1}, f_{2}=g_{1} g_{2}$. Since $\operatorname{deg}_{y}(G(x, y))=p q+r=k$, then $x^{k} G(x, y)=$ $H(x, x y)$, where $\operatorname{deg}_{z}(H(x, z))=k$. Then

$$
g_{1}^{k} G\left(g_{1}, g_{2}\right)=H\left(g_{1}, g_{1} g_{2}\right)=H\left(f_{1}, f_{2}\right),
$$

and consequently,

$$
\operatorname{deg}\left(G\left(g_{1}, g_{2}\right)\right)=\operatorname{deg}\left(H\left(f_{1}, f_{2}\right)\right)-\operatorname{deg}\left(g_{1}^{k}\right) .
$$

Now we show that the elements $f_{1}, f_{2}$ satisfy all the conditions of Theorem 2 . Since $\overline{g_{1}}, \overline{g_{2}}$ are algebraically dependent and $\overline{g_{2}} \notin\left\langle\overline{g_{1}}\right\rangle$, the elements $\overline{f_{1}}=\overline{g_{1}}, \overline{f_{2}}=$ $\overline{g_{1}} \overline{g_{2}}$ are algebraically dependent as well, and $\overline{f_{2}} \notin\left\langle\bar{f}_{1}\right\rangle$. Put $m_{1}=n=\operatorname{deg} f_{1}, m_{2}=$ $n+m=\operatorname{deg} f_{2}$. Since $\operatorname{deg}\left[g_{1}, g_{2}\right] \geq 2$, we have $\operatorname{deg}\left[f_{1}, f_{2}\right]=\operatorname{deg}\left(g_{1}\left[g_{1}, g_{2}\right]\right)=$ $m_{1}+\operatorname{deg}\left[g_{1}, g_{2}\right]>m_{1}$. Thus, all the conditions of Theorem 2 are fulfilled. Note that $\left(m_{1}, m_{2}\right)=(n, n+m)=(n, m)$, and in the notation of Theorem 2 we have 
$m_{0}=\operatorname{deg}\left[g_{1}, g_{2}\right]$. Applying Theorem 2, we obtain from the formula for degree $G\left(g_{1}, g_{2}\right)$ above

$$
\begin{aligned}
\operatorname{deg}\left(G\left(g_{1}, g_{2}\right)\right) & \geq(n+m) k+\left(\operatorname{deg}\left[g_{1}, g_{2}\right]-n-m\right)\left[\frac{k}{p}\right]-n k \\
& =m k-\left(m+n-\operatorname{deg}\left[g_{1}, g_{2}\right]\right) q \\
& =m p q+m r-m q-n q+q \operatorname{deg}\left[g_{1}, g_{2}\right] \\
& =q\left(p m-m-n+\operatorname{deg}\left[g_{1}, g_{2}\right]\right)+m r=q N+m r .
\end{aligned}
$$

Similarly, putting $f_{1}=g_{2}, f_{2}=g_{2} g_{1}$, we get the second part of the theorem.

Evidently, $\frac{m n}{(m, n)} \geq m+n$ and so $N\left(g_{1}, g_{2}\right) \geq \operatorname{deg}\left[g_{1}, g_{2}\right] \geq 2$. Therefore, by [4, 6.9], Theorem 3 immediately implies the theorem of H. Jung [5] on tameness of automorphisms of polynomials in two variables.

Corollary $10([5])$. Every automorphism of a ring of polynomials in two variables over a field of characteristic 0 is tame.

Corollary 11. In the conditions of Theorem 3 , for every $h \in\left\langle g_{1}, g_{2}\right\rangle$, either $\bar{h} \in$ $\left\langle\overline{g_{1}}, \overline{g_{2}}\right\rangle$ or $\operatorname{deg} h \geq N\left(g_{1}, g_{2}\right)$.

Proof. If $h=G\left(g_{1}, g_{2}\right)$ and $\operatorname{deg}_{y}(G(x, y))<p$, then $h$ is a linear combination of the elements of type $g_{1}^{i} g_{2}^{j}, j<p$. By the proof of Lemma 6 , these elements have different degrees for different values of $i, j$. Consequently, $\bar{h}=\alpha \overline{g_{1}}{\overline{g_{2}}}^{j}$ and $\bar{h} \in\left\langle\overline{g_{1}}, \overline{g_{2}}\right\rangle$. If $\operatorname{deg}_{y}(G(x, y)) \geq p$, then in the conditions of Theorem 3 we have $q \geq 1$ and $\operatorname{deg} h \geq N\left(g_{1}, g_{2}\right)$.

Note that if $p>2$ or $\operatorname{deg}\left[g_{1}, g_{2}\right]>n$, then $N\left(g_{1}, g_{2}\right)>m$. We now give some examples of $*$-reduced pairs with the condition $p=2, \operatorname{deg}\left[g_{1}, g_{2}\right] \leq n$.

Example 1. Let $a=x+y^{2}, b=y$, and

$$
\begin{aligned}
& g_{1}=b+a^{2}=y+x^{2}+2 x y^{2}+y^{4}, \\
& g_{2}=3 a b+2 a^{3}=3 x y+2 x^{3}+3 y^{3}+6 x^{2} y^{2}+6 x y^{4}+2 y^{6} .
\end{aligned}
$$

Then $\left[g_{1}, g_{2}\right]=3 b[b, a]=-3 y[x, y], \operatorname{deg} g_{1}=n=4, \operatorname{deg} g_{2}=m=6, \operatorname{deg}\left[g_{1}, g_{2}\right]=$ 3 , and in the notation of Theorem 3 we have $p=2$. The element

$$
f=2 g_{2}^{2}-8 g_{1}^{3}+3 g_{2}=6 x y^{4}+12 x^{2} y^{2}+6 x^{3}+y^{3}+9 x y
$$

has degree $5=N\left(g_{1}, g_{2}\right)<m$, and the lower bound of the estimation of Theorem 3 is accessible.

Example 2. Let $a=x+y^{2}+y^{3}, b=y$, and

$$
\begin{aligned}
g_{1}= & b+a^{2}=y+x^{2}+2 x y^{2}+2 x y^{3}+y^{4}+2 y^{5}+y^{6}, \\
g_{2}= & 3 a b+2 a^{3}=3 x y+2 x^{3}+3 y^{3}+6 x^{2} y^{2}+3 y^{4}+6 x^{2} y^{3}+6 x y^{4} \\
& +12 x y^{5}+2 y^{6}+6 x y^{6}+6 y^{7}+6 y^{8}+2 y^{9} .
\end{aligned}
$$

Then $\left[g_{1}, g_{2}\right]=3 b[b, a]=-3 y[x, y], \operatorname{deg} g_{1}=n=6, \operatorname{deg} g_{2}=m=9, \operatorname{deg}\left[g_{1}, g_{2}\right]=$ 3 , and in the notation of Theorem 3 we have $p=2$. Consider the element

$$
f=4 g_{1}^{3}-g_{2}^{2}=3 y^{8}+6 y^{7}+3 y^{6}+6 x y^{5}+6 x y^{4}+3 x^{2} y^{2}+4 y^{3} .
$$

We have $\operatorname{deg} f=8>N\left(g_{1}, g_{2}\right)=6$, and so the lower bound of the estimation of Theorem 3 is not accessible. 
Problem 2. Does there exist an estimation, as in Theorem 3, for an algebraically dependent reduced pair of elements? In particular, will the estimation of Theorem 3 be true for an algebraically dependent reduced pair of elements if we change $\operatorname{deg}\left[g_{1}, g_{2}\right]$ to 1 ?

Certainly, a positive solution of this question would extend the theorem [1.

Example 3. If we set $x=0$ in Example 1, then we get the algebraically dependent reduced pair of elements

$$
g_{1}=y+y^{4}, g_{2}=3 y^{3}+2 y^{6} .
$$

The subalgebra $\left\langle g_{1}, g_{2}\right\rangle$ contains the element $y^{3}$ of degree 3 .

\section{ACKNOWLEDGMENTS}

The second author is grateful to the Institute of Mathematics and Statistics of the University of Sao Paulo for its warm hospitality during his visit from October 9, 2000 until August 31, 2001 when the main part of this work was done.

We are grateful to L. Makar-Limanov, A. A. Mikhalev, V. Shpilrain and J. -T. $\mathrm{Yu}$ for thoroughly going over the details of the proofs during an informal seminar at the University of Hong Kong in June 2002. We also thank A. van den Essen, D. Wright, and E. Zelmanov for numerous helpful comments.

\section{REFERENCES}

[1] S. S. Abhyankar, T. -T. Moh, Embeddings of the line in the plane, J. reine angew. Math. 276 (1975), 148-166. MR 52:407

[2] H. Bass, E. H. Connell, D. Wright, The Jacobian conjecture: reduction of degree and formal expansion of the inverse, Bull. Amer. Math. Soc. (N. S.) 7 (1982), no. 2, 287-330. MR 83k:14028

[3] G. M. Bergman, Centralizers in free associative algebras, Trans. Amer. Math. Soc. 137 (1969), 327-344. MR 38:4506

[4] P. M. Cohn, Free rings and their relations, 2nd edition, Academic Press, London, 1985. MR 87e:16006

[5] H. W. E. Jung, Über ganze birationale Transformationen der Ebene, J. reine angew. Math. 184 (1942), 161-174. MR 5:74f

[6] L. Makar-Limanov, Locally nilpotent derivations, a new ring invariant and applications, preprint.

[7] G. A. Noskov, The cancelation problem for a ring of polynomials, Sibirsk. Mat. Zh. 19 (1976), no. 6, 1413-1414. MR 81g:13005

[8] A. Nowicki, M. Nagata, Rings of constants for $k$-derivations in $k\left[x_{1}, x_{2}, \ldots, x_{n}\right]$, J. Math. Kyoto Univ. 28-1 (1988), 111-118. MR 89b:13009

[9] D. Shannon, M. Sweedler, Using Gröbner bases to determine algebra membership, split surjective algebra homomorphisms determine birational equivalence, J. Symbolic Comput. 6 (1988), 267-273. MR 90e:13002

[10] I. P. Shestakov, Quantization of Poisson superalgebras and speciality of Jordan Poisson superalgebras, Algebra i logika, 32 (1993), no. 5, 571-584; English translation: in Algebra and Logic, 32 (1993), no. 5, 309-317. MR 95c:17034

[11] U. U. Umirbaev, Universal derivations and subalgebras of free algebras, In Proc. 3rd Internat. Conf. in Algebra (Krasnoyarsk, 1993). Walter de Gruyter, Berlin, 1996, 255-271. MR 97c:16030

[12] J. -T. Yu, On relations between Jacobians and minimal polynomials, Linear Algebra Appl. 221 (1995), 19-29. MR 96c:14014

[13] A. Zaks, Dedekind subrings of $k\left[x_{1}, x_{2}, \ldots, x_{n}\right]$ are rings of polynomials, Israel J. Math. 9 (1971), 285-289. MR 43:6191 
Instituto de Matemática e Estatística, Universidade de São Paulo, Caixa Postal 66281, SÃo Paulo - SP, 05311-970, Brazil; Sobolev Institute of Mathematics, NovosiBIRSK, 630090, RUSSIA

E-mail address: shestak@ime.usp.br

Department of Mathematics, Eurasian National University, Astana, 473021, KazaKHSTAN

E-mail address: umirbaev@yahoo.com 